\title{
Vitamin D and primary hyperparathyroidism: more insights into a complex relationship
}

\author{
Marcella D. Walker ${ }^{1} \cdot$ John P. Bilezikian ${ }^{1}$
}

Received: 13 October 2016 / Accepted: 1 November 2016 / Published online: 17 November 2016

(C) Springer Science+Business Media New York 2016

In primary hyperparathyroidism (PHPT), low levels of vitamin $\mathrm{D}$ are found more often than in the general population [1, 2]. This well established observation is based upon measurement of the serum 25-hydroxyvitamin D level (25OHD). The operational definition of vitamin D deficiency, again based upon 25OHD levels, is viewed by The Institute of Medicine as $<20 \mathrm{ng} / \mathrm{mL}(50 \mathrm{nM} / \mathrm{l})$ [3]. Many experts, however, define two categories of "low" vitamin D: one in which the level is between 20 and $30 \mathrm{ng} / \mathrm{mL}$ (insufficiency) and the other in which the level is $<20 \mathrm{ng} / \mathrm{mL}$ (deficiency). These cut points, while controversial, do not address the special setting of PHPT. The most recent guidelines on the management of asymptomatic PHPT, recommend maintaining or repleting 25OHD to levels $>20$ $\mathrm{ng} / \mathrm{ml}$ [4]. The controversy was acknowledged in that publication, noting that some experts and societies favor a level $>30 \mathrm{ng} / \mathrm{mL}$. These threshold values relate to of the concentration of total $25 \mathrm{OHD}$; that is, the forms that are both protein bound and free. It is the unbound or free $25 \mathrm{OHD}$ that is biologically active, constituting approximately only $1 \%$ of the total concentration. Another small fraction, approximately $10 \%$ is bound to albumin as a complex that is theoretically also biologically available since the binding partition is relatively "loose". The vast majority of circulating $25 \mathrm{OHD}$ is bound to its binding protein, vitamin D binding protein (DBP) and not biologically available. In PHPT, there is limited information

Marcella D. Walker

Mad2037@columbia.edu

1 Department of Medicine, Metabolic Bone Diseases Unit, College of Physicians and Surgeons, Columbia University, New York, NY 10032, USA regarding the relative amounts of these various forms of circulating 25OHD. Whether genetic factors, such as polymorphisms in DBP, affect 25OHD levels in PHPT has not previously been investigated.

The exact pathophysiological mechanism(s) explaining the association between low 25OHD and PHPT are not clear. Several proposed mechanisms are likely to play a role. Parathyroid hormone (PTH) enhances the conversion of $25 \mathrm{OHD}$ to 1,25 -dihydroxyvitamin $\mathrm{D}\left[1,25(\mathrm{OH})_{2} \mathrm{D}\right]$ by inducing the renal 1-alpha hydroxylase enzyme [5]. Increased levels of $1,25(\mathrm{OH})_{2} \mathrm{D}$ in PHPT, in turn, may inhibit the further production of active vitamin $\mathrm{D}$ from precursors in the skin and liver. The half-life of 25OHD may also be shortened in PHPT, with increased metabolic clearance due to enhanced hepatic inactivation [6]. Another view of the pathophysiology of PHPT places chronic 25OHD deficiency as an inciting event leading to parathyroid gland hyperplasia and subsequent adenomatous change.

There is some evidence to suggest that vitamin D deficiency may increase the likelihood of a more symptomatic presentation of PHPT. This idea is supported by data indicating that the clinical and laboratory features of PHPT are more severe in areas of the world where vitamin D deficiency is endemic $[7,8]$. Others have proposed that changes in the clinical presentation of PHPT in the Western world from a highly symptomatic disorder prior to the 1970's to a predominantly asymptomatic one over the last 40 years is due, in part, to higher vitamin D levels resulting from fortification of dairy products with vitamin D during that time or from simply taking vitamin D supplements $[9,10]$. The exact influence of vitamin D status upon the modern presentation of PHPT has yet to be fully defined. 
In this issue of Endocrine, Battista et al. [11] and Viccia et al. [12] report on vitamin D in PHPT and add to our knowledge regarding the pathophysiology of hypovitaminosis D and its role in disease presentation. Battista et al. assessed whether calculated free and bioavailable (free plus albumin-bound) 25OHD levels are low in PHPT and whether vitamin D status is influenced by genetic background [11]. They compared 88 patients with PHPT (mean calcium $11.2 \mathrm{mg} / \mathrm{dl}$, PTH $247 \mathrm{pg} / \mathrm{ml}, 25 \mathrm{OHD} 12.3 \mathrm{ng} / \mathrm{ml}$ ) to matched related and to unrelated family members without PHPT. In addition to confirming prior data indicating that total 25OHD is low in PHPT, they reported that DBP as well as free and bioavailable 25OHD levels are also low in PHPT. While the authors adjusted for several covariates, they lacked information regarding dietary vitamin D intake, sun exposure and weight which are also known to affect $25 \mathrm{OHD}$ levels and could have contributed to the finding of lower 25OHD levels in the PHPT group.

The authors also demonstrate that the frequency of two common DBP single nucleotide polymorphisms or SNPs (rs7041 and 4588) were not more common in PHPT than controls; the SNPs, however, were negatively correlated with DBP levels in all groups, but there was no group-SNP interaction. The authors concluded that 25OHD is low in PHPT compared to controls whether measured as total or calculated free or bioavailable 25OHD. Further, they suggest that while DBP contributes to low vitamin D in PHPT, it alone is not responsible for the hypovitaminosis D and that vitamin D metabolism is likely to be generally disturbed in PHPT. With the limited genetic analysis of 2 SNPs, they suggest further that the etiology of $250 \mathrm{HD}$ deficiency in PHPT is unlikely to be genetic. This study has several important strengths including its relatively large size, the use of comparator groups with and without a shared genetic background, as well as DBP genotype information.

In a separate study, Viccia et al. [12] investigated the association between 25OHD levels and the biochemical and clinical presentation of PHPT in a cross-sectional study of 215 Italian women with mild PHPT (mean calcium $10.5 \mathrm{ng} / \mathrm{ml}$ and PTH $153 \mathrm{pg} / \mathrm{ml}$ ). In addition to assessing biochemistries and bone mineral density (BMD) by vitamin D status, a major strength of the study is that nephrolithiasis and fracture were objectively assessed with renal ultrasound and vertebral fracture assessment, respectively. Mean 25OHD level, as measured by RIA, was in the deficient range (16 $\mathrm{ng} / \mathrm{ml}$ ) with only $7 \%$ having sufficient levels $(\geq 30 \mathrm{ng} / \mathrm{ml})$. As noted by the authors, the RIA assay that was used underestimates $25 \mathrm{OHD}$ concentrations as compared to the gold standard LC-MS/MS assay.

Multiple regression analysis demonstrated independent associations of $25 \mathrm{OHD}$ with biochemical disease severity indices including PTH, bone specific alkaline phosphatase
(BSAP), and estimated glomerular filtration rate (eGFR). Of note, neither BMD by DXA nor serum or urine calcium was associated with the $250 \mathrm{HD}$ level in multiple regression models. In a categorical analysis, the authors found similar results: the lowest quartile of $250 \mathrm{HD}$ was associated with highest PTH level and BSAP as well as lower eGFR. In contrast, serum and urine calcium, fracture incidence, nephrolithiasis and indication for parathyroidectomy were not associated with $25 \mathrm{OHD}$ quartile. Similar results were obtained when groups were classified by common thresholds for vitamin D status. It is unclear, however, if results would have differed if other or more sensitive modalities (abdominal CT and spinal x-rays) were used for the assessment of nephrolithiasis and vertebral fracture, respectively. Based on these results, the authors concluded that low vitamin D was associated with biochemically (PTH and BSAP), but not clinically (stones and fractures), more severe PHPT. These results generally confirm those from a similar study of PHPT patients in New York City [13].

The study has several strengths including its large size, enrollment of patients who were not taking vitamin D supplementation, and use of widely accepted 25OHD thresholds for determining circulating adequacy. The study is limited by the lack of sufficient numbers of subjects who were truly vitamin D sufficient and exclusion of subjects whose serum calcium concentrations were $>12 \mathrm{mg} / \mathrm{dL}$. Thus, the relatively narrow range of vitamin $\mathrm{D}$ and serum calcium concentrations prevented a more comprehensive assessment of how vitamin D inadequacy might contrast with those who are vitamin D sufficient, as well as those whose PHPT is typically associated with symptomatology. The results, nevertheless, do permit an assessment of the potential role of vitamin $\mathrm{D}$ in influencing disease presentation among those whose PHPT is associated with mild hypercalcemia, the most common biochemical phenotype seen in the Western world today. It is also important to interpret the results of Viccia et al. in the context of a recent randomized controlled trial of cholecalciferol in PHPT, which suggests that treatment leads to an increase in lumbar spine BMD without an increase in serum calcium. The study by Viccia et al. and others do not confirm this relationship [13].

The studies by Battista et al. [11] and Viccia et al. [12] add to our understanding of the pathophysiology and effects of hypovitaminosis D in PHPT. The results collectively confirm that 25OHD is low in PHPT (whether measured as total, bioavailable or calculated free). These findings cannot be accounted for by DBP levels or two common DBP polymorphisms alone. Finally, low 25OHD levels influence the biochemical, but not clinical, severity of PHPT in patients with mild disease. Further work is needed to more definitively delineate the pathogenesis of hypovitaminosis D in PHPT as well as optimal levels vitamin D in this disorder. 


\section{Compliance with ethical standards}

Conflict of interest The authors declare that they have no conflict of interest.

\section{References}

1. B. Moosgaard, P. Vestergaard, L. Heickendorff, F. Melsen, P. Christiansen, L. Mosekilde, Vitamin D status, seasonal variations, parathyroid adenoma weight and bone mineral density in primary hyperparathyroidism. Clin. Endocrinol. 63, 506-513 (2005)

2. P. Boudou, F. Ibrahim, C. Cormier, E. Sarfati, J.C. Souberbielle, A very high incidence of low 25 hydroxy-vitamin D serum concentration in a French population of patients with primary hyperparathyroidism. J. Endocrinol. Invest. 29, 511-515 (2006)

3. A.C. Ross, J.E. Manson, S.A. Abrams et al. The 2011 report on dietary reference intakes for calcium and vitamin $\mathrm{D}$ from the Institute of Medicine: what clinicians need to know. J. Clin. Endocrinol. Metab. 96, 53-58 (2011)

4. R. Eastell, M.L. Brandi, A.G. Costa, P. D'Amour, D.M. Shoback, R.V. Thakker, Diagnosis of asymptomatic primary hyperparathyroidism: proceedings of the fourth international workshop. J. Clin. Endocrinol. Metab. 99, 3570-3579 (2014)

5. M.R. Clements, M. Davies, M.E. Hayes et al. The role of 1,25dihydroxyvitamin $\mathrm{D}$ in the mechanism of acquired vitamin $\mathrm{D}$ deficiency. Clin. Endocrinol. 37, 17-27 (1992)
6. M.R. Clements, M. Davies, D.R. Fraser, G.A. Lumb, E.B. Mawer, P.H. Adams, Metabolic inactivation of vitamin D is enhanced in primary hyperparathyroidism. Clin. Sci. 73, 659-664 (1987)

7. J.P. Bilezikian, X. Meng, Y. Shi, S.J. Silverberg, Primary hyperparathyroidism in women: a tale of two cities-New York and Beijing. Int. J. Fertil. Womens Med. 45, 158-165 (2000)

8. J.M. Liu, N.E. Cusano, B.C. Silva et al. Primary hyperparathyroidism: a tale of two cities revisited - New York and Shanghai. Bone Res. 1, 162-169 (2013)

9. D.S. Rao, G. Agarwal, G.B. Talpos et al. Role of vitamin D and calcium nutrition in disease expression and parathyroid tumor growth in primary hyperparathyroidism: a global perspective. J. Bone Miner. Res. 17(Suppl 2), N75-N80 (2002)

10. S.J. Silverberg, Vitamin D deficiency and primary hyperparathyroidism. J. Bone Miner. Res. 22(Suppl 2), V100-V104 (2007)

11. C. Battista, V. Guarnieri, V. Carnevale, et al. Vitamin D status in primary hyperparathyroidism: effect of genetic background. Endocrine 2016. DOI:10.1007/s12020-016-0974-x

12. G. Viccica, F Cetani, E. Vignali, M. Miccoli, C. Marcocci, Impact of vitamin D deficiency on the clinical and biochemical phenotype in women with sporadic primary hyperparathyroidism. Endocrine 2016. DOI:10.1007/s12020-016-0931-8

13. M.D. Walker, E. Cong, J.A. Lee et al. Vitamin D in primary hyperparathyroidism: effects on clinical, biochemical, and densitometric presentation. J. Clin. Endocrinol. Metab. 100, 3443-3451 (2015) 